\title{
Mode excitation by turbulent convection in rotating stars
}

\section{Effect of uniform rotation}

\author{
K. Belkacem ${ }^{1,2}$, S. Mathis ${ }^{3}$, M. J. Goupil², and R. Samadi ${ }^{2}$ \\ ${ }^{1}$ Institut d'Astrophysique et Géophysique, Université de Liège, Allée du 6 Août 17, 4000 Liège, Belgium \\ e-mail: kevin.belkacem@obspm.fr \\ 2 Observatoire de Paris, LESIA, CNRS UMR 8109, 92195 Meudon, France \\ 3 CEA/DSM/IRFU/Service d'Astrophysique, CE Saclay, 91191 Gif-sur-Yvette, France
}

Received 6 April 2009 / Accepted 9 September 2009

\section{ABSTRACT}

\begin{abstract}
We focus on the influence of the Coriolis acceleration on the stochastic excitation of oscillation modes in convective regions of rotating stars. Our aim is to estimate the asymmetry between excitation rates of prograde and retrograde modes. We extend the formalism derived for obtaining stellar $p$ - and $g$-mode amplitudes (Samadi \& Goupil 2001, A\&A, 370, 136; Belkacem et al. 2008, A\&A, 478, 163) to include the effect of the Coriolis acceleration. We then study the special case of uniform rotation for slowly rotating stars by performing a perturbative analysis. This allows us to consider the cases of the Sun and the CoRoT target HD 49933. We find that, in the subsonic regime, the influence of rotation as a direct contribution to mode driving is negligible compared to the Reynolds stress contribution. In slow rotators, the indirect effect of the modification of the eigenfunctions on mode excitation is investigated by performing a perturbative analysis of the excitation rates. The excitation of solar $p$ modes is found to be affected by rotation with excitation-rate asymmetries between prograde and retrograde modes of the order of several percent. Solar low-order $g$ modes are also affected by uniform rotation and their excitation-rate asymmetries are found to reach 10\%. The CoRoT target HD 49933 is rotating more rapidly than the $\operatorname{Sun}\left(\Omega / \Omega_{\odot} \approx 8\right)$, and we show that the resulting excitation-rate asymmetry is about $10 \%$ for the excitation rates of $p$ modes. We demonstrate that $p$ and $g$ mode excitation rates are modified by uniform rotation through the Coriolis acceleration. A study of the effect of differential rotation will be presented in a forthcoming paper.
\end{abstract}

Key words. convection - turbulence - stars: oscillations

\section{Introduction}

Internal dynamical processes in stars and wave excitation, propagation, and induced transport can be strongly influenced by rotation. Those mechanisms modify stellar internal structure and evolution with significant consequences for example for galactic evolution (Maeder 2009). The impact of rotation on stars is now studied by including models of internal transport processes in stellar evolutionary codes (see for instance Talon et al. 1997; Maeder \& Meynet 2000; Espinosa Lara \& Rieutord 2007; Decressin et al. 2009; Maeder 2009, and references therein). Asteroseismology is also being increasingly developed with results from the CoRoT (Michel et al. 2008a,b; Appourchaux et al. 2008) and KEPLER (Christensen-Dalsgaard et al. 2008) missions, which place constraints on stellar modeling. Those spatial missions allow us to study stars that are slow as well as very rapid rotators.

Since the pioneering works of Ulrich (1970) and Leibacher \& Stein (1971), which led to the identification of the solar five-minute oscillations as global acoustic standing waves ( $p$ modes), the Sun internal structure has been determined from the knowledge of its oscillation frequencies. One of the remaining key issues is the detection and identification of gravity modes (Appourchaux et al. 2000; Gabriel et al. 2002; Turck-Chièze et al. 2004; García et al. 2007; Mathur et al. 2007; García et al. 2008a) for determining the rotation profile in the nuclear region
(Mathur et al. 2008; García et al. 2008b). Oscillation modes are indeed crucial for probing the interior of rotating stars.

Stochastic excitation of radial modes by turbulent convection has been investigated by means of several approaches (Goldreich \& Keeley 1977; Goldreich et al. 1994; Balmforth 1992; Samadi \& Goupil 2001; Chaplin et al. 2005). These methods differ from each other in the nature of the assumed excitation sources, the adopted simplifications and approximations, and by the way that the turbulent convection is described (see reviews by Stein et al. 2004; Houdek 2006). Two major mechanisms have nevertheless been identified as driving the resonant $p$ modes of the stellar cavity: the first is related to the Reynolds stress tensor and, as such, represents a mechanical source of excitation; the second is caused by the advection of turbulent fluctuations of entropy by turbulent motions, and as such represents a thermal source of excitation (Goldreich \& Keeley 1977; Samadi \& Goupil 2001). Samadi \& Goupil (2001) proposed a generalized formalism, taking the Reynolds and entropy fluctuation source terms into account. The satisfying agreement between modeling and observational data for the Sun (Belkacem et al. 2006a,b) permitted us to go a step further and investigate the excitation of non-radial modes in the non-rotating case (Belkacem et al. 2008, 2009) and now the effect of rotation.

Our motivation is to investigate the effect of rotation on the mode excitation rates rather than the frequencies. We then focus on the excitation rates of stochastically excited modes for which several issues can be addressed. For example, is the excitation 
rate of a non-axisymmetric mode $(m \neq 0)$ the same as for an axisymmetric one $(m=0)$ ? Are prograde and retrograde modes excited in the same manner and what are the consequences? We pay attention to the Coriolis acceleration effects in stars, neglecting the centrifugal acceleration-induced effects such as star deformation. Our first objective is to determine whether or not uniform rotation can drive the mode efficiently, and our second is to evaluate the excitation-rate asymmetry between prograde and retrograde modes induced by the perturbation of the eigenfunctions by uniform rotation. The effect of differential rotation on the mode excitation rates will be addressed in a forthcoming paper.

The paper is organized as follows. Section 2 introduces the general formalism, and a detailed derivation of the Reynolds, entropy, and rotation-induced source terms is provided. In Sect. 3, the formalism is applied to solar spheroidal modes. The special case of slow rotators, the Sun, and the CoRoT target HD 49933 are then investigated, and the results are discussed. Some conclusions are presented in Sect. 4.

\section{Turbulent stochastic excitation}

\subsection{The inhomogeneous wave equation}

We derive the inhomogeneous wave equation by taking into account the Coriolis acceleration and differential rotation. The fluid velocity field $(\boldsymbol{v})$ is divided into the terms

$\boldsymbol{v}=\boldsymbol{u}+r \sin \theta \Omega(r, \theta) \boldsymbol{e}_{\phi}$,

where $\Omega(r, \theta)$ is the rotational angular frequency assuming an axisymmetric rotation, $r \sin \theta \Omega(r, \theta) \boldsymbol{e}_{\phi}$ is the velocity field associated with rotation, $\boldsymbol{u}$ is the velocity field associated with the turbulent convective motion and waves, and $(r, \theta, \phi)$ are the usual spherical coordinate with their associated unit vector basis $\left\{\boldsymbol{e}_{k}\right\}_{k=\{r, \theta, \phi\}}$. The rotation axis is chosen so as to coincide with the $\theta=0$ axis of the spherical coordinates system of an inertial frame of reference. In this work, meridional circulation is ignored.

The equation of mass conservation and motion in the presence of axisymmetric rotation, can be written as follows (e.g., Unno et al. 1989):

$$
\begin{aligned}
& \frac{\partial \rho}{\partial t}+\nabla \cdot(\rho \boldsymbol{u})=0 \\
& \frac{\partial(\rho \boldsymbol{u})}{\partial t}+\boldsymbol{\nabla}:(\rho \boldsymbol{u} \boldsymbol{u})+\rho\left[\Omega \frac{\partial \boldsymbol{u}}{\partial \phi}+2 \boldsymbol{\Omega} \times \boldsymbol{u}+r \sin \theta \boldsymbol{u} \cdot \boldsymbol{\nabla} \Omega \boldsymbol{e}_{\phi}\right] \\
& \quad=\rho \boldsymbol{g}-\boldsymbol{\nabla} P,
\end{aligned}
$$

where $\boldsymbol{u}$ is the velocity, $\rho$ is the density, $\boldsymbol{\Omega}=\Omega(r, \theta) \boldsymbol{e}_{z}$ is the rotation velocity, $\boldsymbol{e}_{z}$ is the unit vector along the rotation axis, $\boldsymbol{g}$ is the gravitational field, and $P$ is the pressure. We note that the centrifugal force is neglected.

To go further, all physical quantities are divided into an equilibrium one and a perturbation. The subscripts 1 and 0 denote Eulerian perturbations and equilibrium quantities, respectively, except for velocity where the subscript 1 has been dropped for ease of notation. In the following, the velocity field $\boldsymbol{u}$ is divided into two contributions, namely the oscillation velocity $\left(\boldsymbol{v}_{\mathrm{osc}}\right)$ and the turbulent velocity field $\left(\boldsymbol{u}_{\mathrm{t}}\right)$, such that $\boldsymbol{u}=\boldsymbol{v}_{\mathrm{osc}}+\boldsymbol{u}_{\mathrm{t}}$. Then, taking the temporal derivative of the equation of motion and using the mass conservation equation, one then obtains

$$
\left(\frac{\partial^{2}}{\partial t^{2}}-\boldsymbol{L}_{\Omega}\right) \boldsymbol{v}_{\mathrm{osc}}+\boldsymbol{C}_{\mathrm{osc}}=\mathcal{S}_{\mathrm{t}}
$$

where $\boldsymbol{L}_{\Omega}$ is the linear operator that in presence of rotation becomes

$$
\begin{aligned}
& \boldsymbol{L}_{\Omega}=\boldsymbol{\nabla}\left[\alpha_{s} \boldsymbol{v}_{\mathrm{osc}} \cdot \boldsymbol{\nabla} s_{0}+c_{s}^{2} \boldsymbol{\nabla}\left(\rho_{0} \boldsymbol{v}_{\mathrm{osc}}\right)\right]-\boldsymbol{g} \nabla \cdot\left(\rho_{0} \boldsymbol{v}_{\mathrm{osc}}\right) \\
& -\rho_{0} \Omega \frac{\partial^{2} \boldsymbol{v}_{\mathrm{osc}}}{\partial t \partial \phi}-2 \rho_{0} \boldsymbol{\Omega} \times \frac{\partial \boldsymbol{v}_{\mathrm{osc}}}{\partial t}-\rho_{0} r \sin \theta \frac{\partial \boldsymbol{v}_{\mathrm{osc}}}{\partial t} \cdot \boldsymbol{\nabla} \Omega \boldsymbol{e}_{\phi} .
\end{aligned}
$$

The operator $C_{\text {osc }}$ involves both turbulent and pulsational velocities and contributes to the linear dynamical damping (see Samadi \& Goupil 2001, for details):

$$
\begin{aligned}
C_{\mathrm{osc}}= & \frac{\partial}{\partial t}\left[\frac{\partial\left(\rho_{\mathrm{t}} \boldsymbol{v}_{\mathrm{osc}}\right)}{\partial t}+2 \boldsymbol{\nabla}:\left(\rho_{0} \boldsymbol{v}_{\mathrm{osc}} \boldsymbol{u}_{\mathrm{t}}\right)+\rho_{\mathrm{t}} \Omega \frac{\partial \boldsymbol{v}_{\mathrm{osc}}}{\partial \phi}\right. \\
& +2 \rho_{\mathrm{t}} \boldsymbol{\Omega} \times \boldsymbol{v}_{\mathrm{osc}}+\rho_{\mathrm{t}} r \sin \theta\left(\boldsymbol{v}_{\mathrm{osc}} \cdot \boldsymbol{\nabla} \Omega\right) \boldsymbol{e}_{\phi} \\
& \left.+\boldsymbol{\nabla}\left(\alpha_{s} \boldsymbol{v}_{\mathrm{osc}} \cdot \boldsymbol{\nabla} s_{1}+c_{s}^{2} \boldsymbol{\nabla} \cdot\left(\rho_{\mathrm{t}} \boldsymbol{v}_{\mathrm{osc}}\right)\right)\right] .
\end{aligned}
$$

Finally, the $\mathcal{S}_{\mathrm{t}}$ operator that contains the source terms of the inhomogeneous wave equation (Eq. (4)) is given by

$\mathcal{S}_{\mathrm{t}}=-\frac{\partial}{\partial t} \boldsymbol{\nabla}:\left(\rho_{0} \boldsymbol{u}_{\mathrm{t}} \boldsymbol{u}_{\mathrm{t}}\right)+\boldsymbol{\nabla}\left(\alpha_{s} \boldsymbol{u}_{\mathrm{t}} \cdot \boldsymbol{\nabla} s_{\mathrm{t}}\right)+\mathcal{S}_{\Omega}+\mathcal{S}_{M}$

and

$$
\begin{aligned}
\mathcal{S}_{\Omega}= & -\frac{\partial}{\partial t}\left[\rho_{\mathrm{t}}\left(\Omega \frac{\partial}{\partial \phi} \boldsymbol{u}_{\mathrm{t}}-2 \boldsymbol{\Omega} \times \boldsymbol{u}_{\mathrm{t}}-r \sin \theta \boldsymbol{u}_{\mathrm{t}} \cdot \boldsymbol{\nabla} \Omega \boldsymbol{e}_{\phi}\right)\right] \\
\mathcal{S}_{M}= & \frac{\partial}{\partial t}\left(\rho_{\mathrm{t}} \boldsymbol{g}_{1}\right)+\boldsymbol{\nabla}\left[c_{s}^{2} \boldsymbol{\nabla} \cdot\left(\rho_{\mathrm{t}} \boldsymbol{u}_{\mathrm{t}}\right)\right]-\boldsymbol{g} \boldsymbol{\nabla} \cdot\left(\rho_{\mathrm{t}} \boldsymbol{u}_{\mathrm{t}}\right) \\
& -\frac{\partial^{2}}{\partial t^{2}}\left(\rho_{\mathrm{t}} \boldsymbol{u}_{\mathrm{t}}\right)+\mathcal{L}_{\mathrm{t}}
\end{aligned}
$$

where $\boldsymbol{g}_{1}$ is the perturbation of the gravitational acceleration. The first two terms of Eq. (7) correspond to the Reynolds stress and entropy contributions, respectively. The three following terms are contributions associated with rotation. Eventually, as shown by Samadi \& Goupil (2001), the terms in $\mathcal{S}_{M}$ do not contribute significantly to the excitation and are thus neglected as well as the linear terms ${ }^{1}\left(\mathcal{L}_{\mathrm{t}}\right)$.

\subsection{Mean square amplitude for uniform rotation}

Using Eq. (4), the next step is to determine the mean square amplitude of $\boldsymbol{v}_{\text {osc }}$. The procedure is the same as described in Belkacem et al. (2008). The wave velocity field is related to the displacement by means of the relation (Unno et al. 1989)

$\boldsymbol{v}_{\mathrm{osc}}=A\left[i \sigma \boldsymbol{\xi}-(\boldsymbol{\xi} \cdot \boldsymbol{\nabla} \Omega) r \sin \theta \boldsymbol{e}_{\phi}\right] \mathrm{e}^{\mathrm{i} \sigma t}$,

where $\sigma=\omega_{0}+m \Omega$, and $\omega_{0}$ is the mode frequency without rotation. For uniform rotation, it reduces to

$\boldsymbol{v}_{\mathrm{osc}}=A i \sigma \boldsymbol{\xi} \mathrm{e}^{\mathrm{i} \sigma t}$

where $\sigma$ is the eigenfrequency, $\boldsymbol{\xi}(\boldsymbol{r})$ is the displacement eigenfunction in absence of turbulence, and $A(t)$ is the amplitude due to the turbulent forcing. In the presence of rotation, the wave displacement $(\boldsymbol{\xi})$ is expressed as

$$
\begin{aligned}
\boldsymbol{\xi}(\boldsymbol{r})= & \sum_{\ell, m}\left[\xi_{\mathrm{r}}^{\ell, m} \boldsymbol{e}_{\mathrm{r}} Y_{\ell, m}+\left(\xi_{\mathrm{H}}^{\ell, m} \frac{\partial Y_{\ell, m}}{\partial \theta}+\xi_{\mathrm{T}}^{\ell, m} \frac{1}{\sin \theta} \frac{\partial Y_{\ell, m}}{\partial \phi}\right) \boldsymbol{e}_{\mathrm{H}}\right. \\
& \left.+\left(\xi_{\mathrm{H}}^{\ell, m} \frac{1}{\sin \theta} \frac{\partial Y_{\ell, m}}{\partial \phi}+\xi_{\mathrm{T}}^{\ell, m} \frac{\partial Y_{\ell, m}}{\partial \theta}\right) \boldsymbol{e}_{\mathrm{T}}\right]
\end{aligned}
$$

\footnotetext{
${ }_{1}$ Linear terms are defined as the product of an equilibrium quantity and a fluctuating one.
} 
where $\xi_{\mathrm{r}}, \xi_{\mathrm{H}}$, and $\xi_{\mathrm{T}}$ are the radial, horizontal, and toroidal components of the displacement eigenfunction, respectively. Note that in the following we do not use the upper-scripts ${ }^{\ell, m}$ on the eigenfunction components for ease of notation. Each mode is also labelled with a radial order $n$, which we also omit.

The power $(P)$ injected into each mode with given $(n, \ell, m)$ is then related to the mean-squared amplitude $\left(\left\langle|A|^{2}\right\rangle\right)$ by

$P=\eta\left\langle|A|^{2}\right\rangle I \sigma^{2}$,

where the operator \langle\rangle denotes a statistical average performed on an infinite number of independent realizations, $\eta$ is the damping rate, and $I$ is the mode inertia.

Following Samadi \& Goupil (2001) and Belkacem et al. (2008), one then obtains the mean square amplitude for each mode as

$\left\langle|A|^{2}\right\rangle=\frac{1}{8 \eta(\sigma I)^{2}}\left(C_{\mathrm{R}}^{2}+C_{S}^{2}+C_{\Omega}^{2}+C_{\mathrm{c}}\right)$,

where $C_{\mathrm{R}}^{2}$ is the Reynolds stress contribution, $C_{\Omega}^{2}$ contains the contributions related to the Coriolis acceleration, the Doppler term, and one related to the differential rotation, $C_{S}^{2}$ corresponds to entropy fluctuation contributions, while $C_{\mathrm{c}}$ represents the cross-source terms, i.e., the interferences between the different source terms.

\subsection{Reynolds stress contribution}

Following the formalism of Belkacem et al. (2008), we develop the turbulent Reynolds contribution (see Appendix A for a detailed derivation), which becomes for a given $(\ell, m)$

$C_{\mathrm{R}}^{2}=16 \pi^{4} \int \mathrm{d} r r^{2} \rho_{0} R(r) S_{R}(\sigma)$,

and

$$
\begin{aligned}
R(r)= & \frac{16}{15}\left|\frac{\mathrm{d} \xi_{\mathrm{r}}}{\mathrm{d} r}\right|^{2}+\frac{44}{15}\left|\frac{\xi_{\mathrm{r}}}{r}\right|^{2}+\frac{4}{5}\left(\frac{\xi_{\mathrm{r}}^{*}}{r} \frac{\mathrm{d} \xi_{\mathrm{r}}}{\mathrm{d} r}+\text { c.c. }\right) \\
& +L^{2}\left[\frac{11}{15}\left(|\mathcal{A}|^{2}+|\mathcal{B}|^{2}\right)-\frac{22}{15}\left(\frac{\xi_{\mathrm{r}}^{*} \xi_{\mathrm{H}}}{r^{2}}+\text { c.c. }\right)\right] \\
& +\left|\frac{\xi_{\mathrm{H}}}{r}\right|^{2}\left(\frac{16}{15} L^{4}+\frac{8}{5} \mathcal{F}_{\ell,|m|}-\frac{2}{3} L^{2}\right) \\
& +\left|\frac{\xi_{\mathrm{T}}}{r}\right|^{2}\left(\frac{11}{5} L^{2}\left(L^{2}-2\right)-\frac{8}{5} \mathcal{F}_{\ell,|m|}-\frac{2}{3} L^{2}\right) \\
& -\frac{2}{5} L^{2}\left(\frac{\mathrm{d} \xi_{\mathrm{r}}^{*}}{\mathrm{~d} r} \frac{\xi_{\mathrm{H}}}{r}+\text { c.c. }\right),
\end{aligned}
$$

where

$\mathcal{A}=\frac{\mathrm{d} \xi_{\mathrm{H}}}{\mathrm{d} r}+\frac{1}{r}\left(\xi_{\mathrm{r}}-\xi_{\mathrm{H}}\right) \quad$ and $\quad \mathcal{B}=\frac{\mathrm{d} \xi_{\mathrm{T}}}{\mathrm{d} r}-\frac{\xi_{\mathrm{T}}}{r}$,

and

$\mathcal{F}_{\ell,|m|}=\frac{|m|(2 \ell+1)}{2}\left[\ell(\ell+1)-\left(m^{2}+1\right)\right]$

and $L^{2}=l(l+1)$. Furthermore,

$S_{R}(\sigma)=\int \frac{\mathrm{d} k}{k^{2}} E^{2}(k) \int \mathrm{d} \omega \chi_{k}(\omega+\sigma) \chi_{k}(\omega)$,

where $(\boldsymbol{k}, \omega)$ are the wave number and frequency of the turbulent eddies, and $E(\boldsymbol{k}, \omega)$ is the turbulent kinetic energy spectrum, which is expressed as the product $E(\boldsymbol{k}) \chi_{k}(\omega)$ for isotropic turbulence (Stein 1967). A detailed discussion of the temporal correlation function $\left(\chi_{k}\right)$ is addressed in Samadi et al. (2003).

Note that in absence of rotation (i.e., $\Omega=0$ ), the toroidal component of the eigenfunction $\xi_{\mathrm{T}}$ vanishes in Eq. (12) and for $C_{\mathrm{R}}^{2}$ and $R(r)$ we recover the expressions given by Eqs. (22) and (23) of Belkacem et al. (2008). From Eq. (16), additional terms are found to appear through the toroidal component of the eigenfunction. All are found to be positive regardless of $\ell$ and $m$, implying an increase in the excitation rates.

We emphasize that rotation is understood to create anisotropies in the Reynolds stress tensor, then off-diagonal terms (e.g., Kumar et al. 1995; Miesch 2005). An adapted spectral description of turbulent convection including the effect of rotation is thus required to compute Eq. (19) and is beyong the scope or our study.

\subsection{Entropy fluctuation contribution}

As shown by Samadi \& Goupil (2001) and Belkacem et al. (2006b), the Reynolds stress contribution is not the unique source of excitation but one has to account for the excitation by the entropy contribution to reproduce the excitation rates for solar radial $p$ modes.

Following Belkacem et al. (2008), the entropy source term depends on the mode compressibility that can be estimated as

$\int_{\bar{\Omega}} \mathrm{d} \bar{\Omega} Y_{\ell}^{m} \boldsymbol{\nabla} \cdot \boldsymbol{\xi}=\frac{1}{r^{2}} \frac{\mathrm{d}}{\mathrm{d} r}\left(r^{2} \xi_{\mathrm{r}}\right)-\frac{L^{2}}{r} \xi_{\mathrm{H}}$,

where $\bar{\Omega}$ is the solid angle, and the spherical harmonics are normalized following Eq. (A.2).

Hence, from Eqs. (12) and (20) the divergence of the toroidal component, which is the curl of the spherical harmonic, vanishes. Consequently, one obtains the same result as for taking only the poloidal contribution into account. The final expression for the contribution of entropy fluctuations remains the same as in Belkacem et al. (2008), i.e.,

$C_{S}^{2}=\frac{4 \pi^{3} \mathcal{H}}{\sigma^{2}} \int \mathrm{d}^{3} x_{0} \alpha_{s}^{2}\left(A_{\ell}+B_{\ell}\right) \mathcal{S}_{S}(\sigma)$,

where $\mathcal{H}$ is the anisotropy factor introduced in Samadi \& Goupil (2001), which, for the current assumption (isotropic turbulence), is equal to $4 / 3$. In addition,

$$
\begin{aligned}
A_{\ell} & \equiv \frac{1}{r^{2}}\left|D_{\ell} \frac{\mathrm{d}\left(\ln \left|\alpha_{s}\right|\right)}{\mathrm{d} \ln r}-\frac{\mathrm{d} D_{\ell}}{\mathrm{d} \ln r}\right|^{2}, \\
B_{\ell} & \equiv \frac{1}{r^{2}} L^{2}\left|D_{\ell}\right|^{2}, \\
D_{\ell} & =\frac{1}{r^{2}} \frac{\mathrm{d}}{\mathrm{d} r}\left(r^{2} \xi_{\mathrm{r}}\right)-\frac{L^{2}}{r} \xi_{\mathrm{H}}
\end{aligned}
$$

where

$\mathcal{S}_{S}(\sigma) \equiv \int \frac{\mathrm{d} k}{k^{4}} E(k) E_{S}(k) \int \mathrm{d} \omega \chi_{k}(\sigma+\omega) \chi_{k}(\omega)$.

In contrast to the Reynolds contribution expression Eq. (16), the entropy one is not directly modified by rotation. Nevertheless, this contribution can be influenced indirectly by means of the modification of the radial and horizontal components of the eigenfunctions $\left(\xi_{\mathrm{r}}\right.$ and $\xi_{\mathrm{H}}$ ) by the Coriolis acceleration. 


\subsection{Rotational contributions}

The rotational contributions in the inhomogeneous equation (Eqs. (4) and (8)) are

- the contribution related to the Coriolis acceleration

$$
-\frac{\partial}{\partial t}\left(2 \rho_{\mathrm{t}} \boldsymbol{\Omega} \times \boldsymbol{u}_{\mathrm{t}}\right)=-2 \boldsymbol{\Omega} \times \frac{\partial}{\partial t}\left(\rho_{\mathrm{t}} \boldsymbol{u}_{\mathrm{t}}\right)
$$

where we neglect the time variations in the angular velocity on a dynamical time scale.

- the contribution related to the Doppler shift

$$
\frac{\partial}{\partial t}\left(\rho_{\mathrm{t}} \Omega \frac{\partial \boldsymbol{u}_{\mathrm{t}}}{\partial \phi}\right)=\Omega \frac{\partial}{\partial t}\left(\rho_{\mathrm{t}} \frac{\partial \boldsymbol{u}_{\mathrm{t}}}{\partial \phi}\right),
$$

- the contribution related to the differential rotation

$$
-\frac{\partial}{\partial t}\left(\rho_{\mathrm{t}} r \sin \theta \boldsymbol{u}_{\mathrm{t}} \cdot \boldsymbol{\nabla} \Omega\right) \boldsymbol{e}_{\phi}=r \sin \theta \frac{\partial \rho_{\mathrm{t}} \boldsymbol{u}_{\mathrm{t}}}{\partial t} \cdot \boldsymbol{\nabla} \Omega \boldsymbol{e}_{\phi}
$$

In this paper, we consider only uniform rotation, hence the last contribution (Eq. (28)) vanishes. Nevertheless, all contributions, i.e., from Eqs. (26) to (28), are proportional to the perturbed mass flux $\rho_{\mathrm{t}} \boldsymbol{u}_{\mathrm{t}}$. A dimensional analysis (see Samadi \& Goupil 2001, for details) shows that all those terms then scale as the Mach number to the third $\left(\mathcal{M}^{3}\right)$. Compared to the Reynolds contribution, which scales as $\mathcal{M}^{2}$, all rotational contributions are negligible in the subsonic regime. For the Sun, this conclusion remains valid even for the uppermost layers where $\mathcal{M} \approx 0.3$. In addition, the rotational velocity appears from Eq. (26) to Eq. (28) introducing the ratio $\Omega / \sigma$, which is very small for slow rotators.

Eventually, one obtains (see Eq. (15), Eq. (21), and Eqs. (26) to $(27))$

$$
\begin{aligned}
C_{\mathrm{R}}^{2} & =O\left(\mathcal{M}^{4}\right) \gg C_{S}^{2}=O\left(\mathcal{M}^{6}\right) \gg C_{\mathrm{R} \Omega}=O\left(\mathcal{M}^{5}\right)\left(\frac{\Omega}{\sigma}\right) \\
& \gg C_{\Omega}^{2}=O\left(\mathcal{M}^{6}\right) O\left(\frac{\Omega}{\sigma}\right)^{2},
\end{aligned}
$$

where $C_{\mathrm{R} \Omega}$ is the coupled source term associated with the Reynolds stress and rotational contributions. Consequently, in the following only the Reynolds stress contribution will be considered.

\subsection{Final balance}

We have shown in Sects. 2.3 to 2.5 that in the presence of uniform rotation, the Reynolds term contribution (Eq. (15)) remains the most dominant in the subsonic regime. It can be influenced by uniform rotation in three ways;

- the turbulent velocity field can be modified by the Coriolis acceleration, hence affecting the Reynolds contribution in Eq. (15) by the source term (Eq. (19));

- the toroidal component of the eigenfunction introduces additional terms in Eq. (16);

- eventually, the poloidal components of the eigenfunctions are modified by the Coriolis acceleration and will influence the Reynolds contribution in terms of Eq. (15).

\section{Application to spheroidal modes of slow rotators}

As mentioned in Sect. 2.6, the velocity field can be modified by the Coriolis acceleration. However, for slow rotators the rotation rate does not significantly affect the turbulent field in the upper convective region where modes are excited, provided that the ratio of the convective frequency to the rotation rate is higher than unity. For the Sun, this requirement is fulfilled in the entire convective region except in the deepest layers, near the interface with the radiative region. Nevertheless, the contribution of these deep layers do not contribute significantly to the excitation rates for the modes considered here, i.e., low-order $g$ modes and $p$ modes. Hence, in the following we assume that the turbulent field, and its spectral dependence, are not affected by uniform rotation. Note, however, that for lower frequencies, and especially asymptotic gravity-modes, this approximation is no longer valid since a significant contribution to the mode excitation comes from the deeper convective layers (Belkacem et al. 2009).

We then consider the effect of the perturbation of the mode excitation rates by the Coriolis acceleration associated with the modification of the eigenfunctions. In this framework, we use a perturbative approach, which is valid for slow rotators and particularly for the Sun since we restrict our investigation to rather high-frequency $p$ and $g$ modes. The ratio of the mode frequency to the rotation rate is still higher than unity. In the Sun, for a typical $p$ mode at $v=3 \mathrm{mHz}$, one has $\omega_{0} / 2 \Omega \approx 3 \times 10^{3} \gg 1$ in the convective region where modes are excited, and for a solar $g$ mode at $v=100 \mu \mathrm{Hz}$, this ratio remains high at $\omega_{0} / 2 \Omega \approx$ $100 \gg 1$. This allows us to use a perturbative approach.

\subsection{Perturbation of the mode excitation rates}

Our aim is to derive an analytical expression of excitation rates influenced by uniform rotation using a perturbative method. Following the classical method given in Unno et al. (1989), we develop the eigenfunction for a given $n, \ell, m$ to first order

$$
\begin{aligned}
& \xi_{\mathrm{r}}=\xi_{\mathrm{r} ; n, \ell}^{(0)}+m\left(\frac{2 \Omega}{\omega_{0}}\right) \sum_{n^{\prime} \neq n} C_{n^{\prime}, n, \ell} \xi_{\mathrm{r} ; n^{\prime}, \ell}^{(0)}, \\
& \xi_{\mathrm{H}}=\xi_{\mathrm{H} ; n, \ell}^{(0)}+m\left(\frac{2 \Omega}{\omega_{0}}\right) \sum_{n^{\prime} \neq n} C_{n^{\prime}, n, \ell} \xi_{\mathrm{H} ; n^{\prime}, \ell^{\prime}}^{(0)},
\end{aligned}
$$

and for the toroidal part

$$
\begin{aligned}
\xi_{\mathrm{T}}= & i\left(\frac{2 \Omega}{\omega_{0}}\right)\left\{\ell D_{\ell, m}\left[\xi_{\mathrm{r} ; n, \ell-1}^{(0)}-(\ell-1) \xi_{\mathrm{H} ; n, \ell-1}^{(0)}\right]\right. \\
& \left.-(\ell+1) D_{\ell+1, m}\left[\xi_{\mathrm{r} ; n, \ell+1}^{(0)}+(\ell+2) \xi_{\mathrm{H} ; n, \ell+1}^{(0)}\right]\right\},
\end{aligned}
$$

where

$D_{\ell, m}=\frac{1}{\ell^{2}} \sqrt{\frac{\ell^{2}-m^{2}}{4 \ell^{2}-1}}$.

The expression for $C_{n^{\prime}, n, \ell}$ is given in Appendix B, and $\xi_{\mathrm{r} ; n, \ell}^{(0)}, \xi_{\mathrm{H} ; n, \ell}^{(0)}$ are the radial and horizontal components of the eigenfunction in absence of rotation.

As pointed out by Dziembowski \& Goode (1992), the convergence properties of the sum involved in Eq. (B.1) and Eq. (B.2) are unclear. As shown by Eq. (B.4), this is particularly problematic for a dense spectrum such as high-order gravity modes in the Sun. We nevertheless use it for convenience. An alternative exists (Dziembowski \& Goode 1992), which consists of computing a modified eigenvalue problem. However, this second possibility makes it more difficult to identify contributions to the excitation rates. 
Inserting the decomposition (Eqs. (B.1) to (B.3)) into Eqs. (15) and (16), we obtain (see Appendix B for the detailed calculation)

$P_{m}=P^{(0)}+m\left(\frac{2 \Omega}{\omega_{0}}\right) P_{|m|}^{(1)}$,

where

$P^{(0)}=\frac{4 \pi^{3}}{8 I} \int \mathrm{d} m R^{(0)} S_{R}\left(\omega_{0}\right)$,

$P_{|m|}^{(1)}=\frac{4 \pi^{3}}{8 I} \int \mathrm{d} m R_{|m|}^{(1)} S_{R}\left(\omega_{0}\right)$,

and $R^{(0)}$ and $R^{(1)}$ corresponds to the perturbative expansion of $R$ (Eq. (16)) given in Appendix B. Note that the zeroth-order terms $\left({ }^{0}\right)$ correspond to the case without rotation. Only the first order in $(2 \Omega / \sigma)$ is considered. Accordingly, the contributions of $\xi_{\mathrm{T}}$ in Eq. (15) are neglected because they are of second order.

We now define the excitation rates asymmetry, between prograde and retrograde modes to first order such as

$\frac{\delta P_{m}}{P_{m}}=\frac{P_{|m|}-P_{-|m|}}{P_{|m|}} \approx 2 m\left(\frac{2 \Omega}{\omega_{0}}\right)\left(\frac{P_{|m|}^{(1)}}{P^{(0)}}\right)$.

From Eq. (37), two factors contribute to the asymmetry namely the ratio $\left(2 \Omega / \omega_{0}\right)$ and $P_{|m|}^{(1)} / P^{(0)}$. They are discussed in the following sections.

\subsection{Application to slow rotators}

\subsubsection{The Sun}

Using the same numerical computation as described in Belkacem et al. (2008), we apply this formalism (Eqs. (34)-(36)) to the solar case. Figure 1 displays the mode excitation rates for the $\ell=1, p$ and $g$ modes. It also presents the ratio $\delta P_{m} / P_{m}$, defined in Eq. (37), which emphasizes the effect of the mode excitation rate asymmetry between the prograde and retrograde modes.

It turns out that the excitation rates of acoustic modes are modified with an excitation rate asymmetry of the order of the percent, which increases toward $g$ modes. We find that the variation in the mode excitation-rate asymmetry with frequency is caused by the term $2 \Omega / \omega_{0}$ in Eq. (37), while the ratio $P_{|m|}^{(1)} / P^{(0)}$ remains of the order of the value of one. $P_{|m|}^{(1)}$ is dominated by the first term in Eq. (B.12), which corresponds to the contribution of the radial component of the eigenfunction, for $p$ modes. For $g$ modes, the horizontal component of the eigenfunction is also of importance and contributes significantly to $P_{|m|}^{(1)}$.

For higher values of the angular degree $(\ell)$, as shown by Fig. 2, there are two effects. First, the higher the azimuthal order $m$, the higher the mode excitation rates asymmetry, at fixed $\ell$. This is explained by the perturbation of the mode excitation rates being proportional to $m$ in Eq. (34). Second, at fixed $m$, the higher the angular degree, the lower the mode excitationrate asymmetry. This behavior comes from the frequency shift of high- $\ell$ modes, since at fixed radial order, the higher the angular degree the higher the mode frequency. Hence, for the same radial order the ratio $2 \Omega / \omega_{0}$ will decrease with the angular degree explaining the behavior in Fig. 2 .
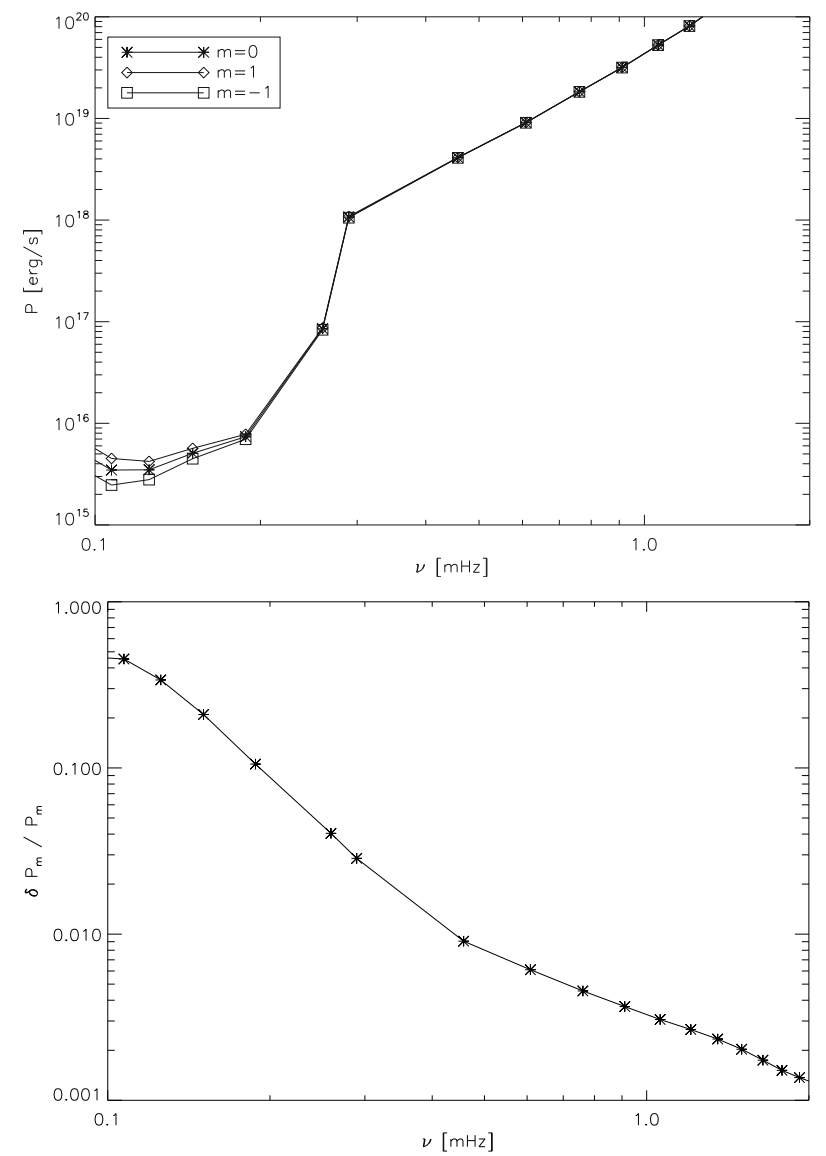

Fig. 1. Top: excitation rates for the mode $\ell=1$ and $m=\{-1,0,1\}$, computed using Eqs. (13)-(15) and using the same solar model as detailed in Belkacem et al. (2008). The depression in the excitation rates at $v \approx 300 \mu \mathrm{Hz}$ is associated with the mixed nature of the modes that consequently produce a higher inertia thus a lower P. Bottom: bias between prograde and retrograde modes, defined in Eq. (37), for the same modes as for the figure in the top panel.

\subsubsection{The CoRoT target HD 49933}

We now consider more rapid rotators, such as HD 49933. This is an F5 V main-sequence star observed twice by the CoRoT mission $^{2}$, first during 62 days and more recently for more than 150 days. The unprecedented photometric precision achieved by the CoRoT mission (Michel et al. 2008a; Auvergne et al. 2009) makes this star a good candidate for the detection of mode excitation-rate asymmetry, which requires, as previously mentioned, accurate measurements. This star, indeed, exhibits a surface rotation period that is shorter than that of the Sun, $P_{\Omega} \approx 3.4$ days (i.e., $\Omega / \Omega_{\odot} \approx 8$ ) as shown by Appourchaux et al. (2008), but still slow enough to ensure that the perturbative approach is valid.

In Fig. 3, we present the same ratio as in Fig. 2 for the $\ell=1$, $p$ modes using a model of HD 49933 that matches the seismic constraints derived by Appourchaux et al. (2008) (Goupil et al. 2009). The asymmetry between the excitation rates of $m=1$ and $m=-1$ modes is found to reach up to $10 \%$. In terms of mode excitation-rate asymmetry, the differences between the Sun and HD 49933 is due to a higher ratio $\left(2 \Omega / \omega_{0}\right)$ in Eq. (37).

2 The CoRoT space mission, launched on December 27th 2006, has been developed and is operated by CNES, with the contribution of Austria, Belgium, Brazil, ESA (RSSD and Science Program), Germany and Spain. 


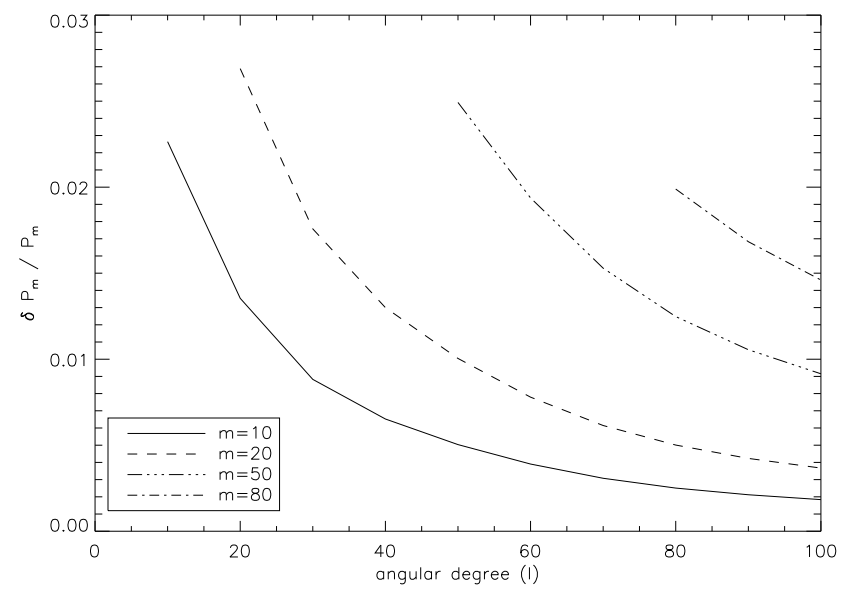

Fig. 2. Bias between prograde and retrograde modes, defined in Eq. (37), for four values of the azimuthal order $m$ and the radial order $n=5$ as a function of the angular degree $\ell$. The computations are performed in the same manner as for Fig. 1.

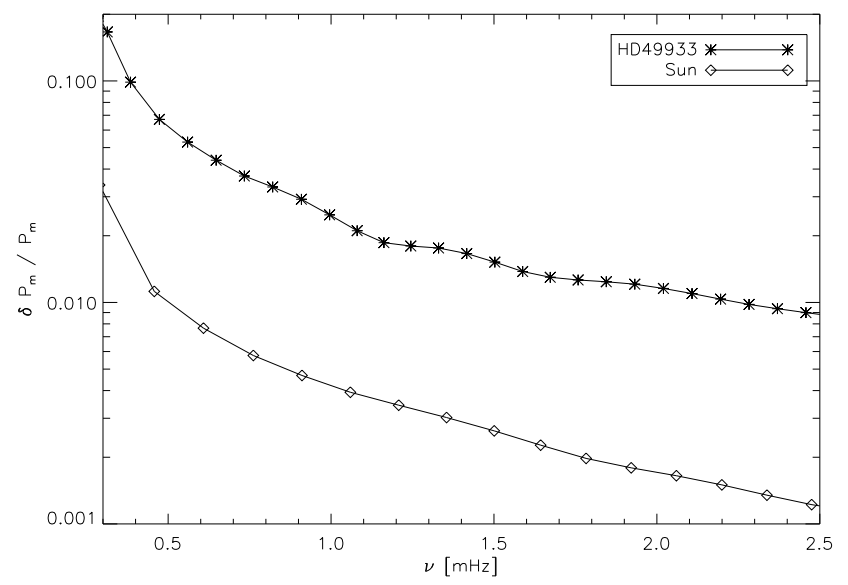

Fig. 3. Bias between prograde and retrograde modes, for the $\ell=1$ modes of the star HD $49933\left(P_{\Omega}=3.4 \mathrm{~d}\right)$ and the $\operatorname{Sun}\left(P_{\Omega}=28 \mathrm{~d}\right)$.

This demonstrates that an asymmetry in terms of mode excitation rates is more likely to be observable for more rapid rotators than the Sun, even if, in contrast to the Sun, only low- $\ell$ modes are observed.

\section{Conclusion and perspectives}

\subsection{Conclusion}

We have derived a formalism that models the stochastic excitation of oscillation modes by convective motions in uniformly rotating stellar regions. We have shown that the driving terms, due to rotation, that appear in the inhomogeneous wave equation are negligible with respect to the Reynolds stress contribution. We demonstrate that the dominant contribution to the excitation rates then comes from the modification of the eigenfunction by the Coriolis acceleration.

The formalism is then applied to low-order $g$ modes and $p$ modes of slow rotators, and in particular the Sun and the CoRoT target HD 49933. For the Sun, a bias between pro- and retrograde waves is found in the excitation rates. For $g$ modes, this bias can reach values of up to $10 \%$. For low- $\ell p$ modes, this bias is found to be of the order of a percent. The detection of the mode excitation-rate asymmetry of individual $p$ modes is not yet possible since the observational error bars obtained, for instance by GOLF, are around 20\% (Belkacem et al. 2006b), while we search for a physical effect of only several percent.

For more rapid rotators, such as HD49933, we find that the excitation-rate asymmetry of low $\ell(\ell=1) p$ modes can reach up to $10 \%$. However, this value is achieved at low frequency, where seismic measurements are generally dominated by the granulation background. In the case of the CoRoT target HD 49933, detection of acoustic modes is limited to the frequency domain $v \in[1.2 ; 2.5] \mathrm{mHz}$. In this frequency domain, the asymmetry in $P$ is no greater than $2 \%$. In contrast, the 1- $\sigma$ uncertainties associated with $P$ are in the range $30 \%-80 \%$, depending on the frequency, for the observations completed during the CoRoT initial run (Appourchaux et al. 2008; Samadi et al. 2009). For the second set of observations of HD 49933 by CoRoT, the $1-\sigma$ uncertainties associated with $P$ is expected to be lower, i.e., in the range 20\%-40\% (Benomar, private communication). Furthermore, current seismic analyses (e.g., Appourchaux et al. 2008) do not reproduce individual mode multiplets but assume a fixed amplitude ratio of the different mode multiplets or even assume a fixed amplitude ratio of the different $\ell$ degree. Therefore, despite the high precision of the CoRoT instrument, it is presently not possible to constraint $P$ for an individual mode multiplet (i.e., for a given value of $\ell$ and $m$ ). Concerning the Kepler instrument, its performance in terms of photon noise level is expected to be a factor five lower in terms of power compared to that achieved for the brightest stars of the CoRoT mission (Chaplin et al. 2008). On the other hand, Kepler will observe the seismic targets over a much longer period (around 4 years) than the CoRoT mission, which we hope will permit us to constrain individual mode multiplets. Another way to proceed is to consider a sum of $P$ for a given $m$ so as to reduce the actual observational errorbars in both the Solar case and that of HD49933.

We note that mode amplitude is a balance between driving and damping. Therefore, asymmetries in mode amplitudes cannot be inferred only from excitation rates since some possible asymmetry in the mode damping rate can arise. This is not investigated here but left to future work.

\subsection{Perspectives}

The effect of uniform rotation on the mode amplitude excitation rates presented here is exploratory work that requires further investigation and theoretical developments.

Stellar convection zones are differentially rotating. Therefore, the next step would be to take the differential rotation into account in both the radial and the latitudinal directions. In contrast to uniform rotation, a consideration at Eq. (10) permits us to understand that the driving source terms in the inhomogeneous wave equation Eq. (4) are modified by differential rotation. The effect of differential rotation on mode excitation rates is the scope of an upcoming paper.

The regime of rapid rotation should also be addressed. The formalism must be adapted to the specific geometry of those stars, since spherical coordinates become inappropriate and one may have to take the star deformation into account. In addition, the eigenfunctions and frequencies have to be derived from an adapted non-perturbative method (e.g., Reese et al. 2006) since rotation can strongly modify both the eigenfunctions and the stellar structure of a star (Rieutord \& Valdettaro 1997; Dintrans \& Rieutord 2000; Reese et al. 2006). Furthermore, in such a regime new types of waves appear that deserve a further study, such as inertial waves and gravito-inertial waves (Rieutord \& Valdettaro 1997; Dintrans \& Rieutord 2000; Mathis et al. 2008). 
In addition, in contrast to slow rotators, the turbulent field is also affected by rotation and the spectral description of turbulence must be taken it into account. The assumption of isotropic turbulence is should then be excluded and the spectral properties of the turbulent field be specified. Numerical simulations can be of some help. As done for solar $g$ modes using the ASH code (Belkacem et al. 2009), it is possible to assess the turbulent properties of these rotators from numerical simulations.

Acknowledgements. K.B. acknowledges financial support from Liège University through the subside fédéral pour la recherche.

\section{Appendix A: Detailed expressions for the Reynolds source term}

The eigenfunctions $(\boldsymbol{\xi})$ are developed in spherical coordinates $\left(\boldsymbol{e}_{\mathrm{r}}, \boldsymbol{e}_{\theta}, \boldsymbol{e}_{\phi}\right)$ and expanded as a sum over spherical harmonics. Hence the fluid displacement eigenfunction for a mode with given $\ell, m$ is written as

$$
\begin{aligned}
\boldsymbol{\xi}(\boldsymbol{r})= & \sum_{\ell, m}\left[\xi_{\mathrm{r} ; \ell, m} \boldsymbol{e}_{\mathrm{r}} Y_{\ell, m}+\left(\xi_{\mathrm{H} ; \ell, m} \frac{\partial Y_{\ell, m}}{\partial \theta}+\xi_{T ; \ell, m} \frac{1}{\sin \theta} \frac{\partial Y_{\ell, m}}{\partial \phi}\right) \boldsymbol{e}_{\mathrm{H}}\right. \\
& \left.+\left(\xi_{\mathrm{H} ; \ell, m} \frac{1}{\sin \theta} \frac{\partial Y_{\ell, m}}{\partial \phi}+\xi_{T ; \ell, m} \frac{\partial Y_{\ell, m}}{\partial \theta}\right) \boldsymbol{e}_{\mathrm{T}}\right]
\end{aligned}
$$

where the spherical harmonics $\left(Y_{\ell, m}(\theta, \phi)\right)$ are normalized according to

$$
\int \frac{d \bar{\Omega}}{4 \pi} Y_{\ell, m} Y_{\ell, m}^{*}=1
$$

with $\bar{\Omega}$ being the solid angle $(\mathrm{d} \bar{\Omega}=\sin \theta \mathrm{d} \theta \mathrm{d} \phi)$.

The Reynolds stress contribution can be written as (see Belkacem et al. 2008, for details)

$$
\begin{aligned}
C_{\mathrm{R}}^{2}= & \pi^{2} \int \mathrm{d}^{3} x_{0}\left(\rho_{0}^{2} b_{i j}^{*} b_{l m}\right) \int \mathrm{d}^{3} k \int \mathrm{d} \omega \\
& \times\left(T^{i j l m}+T^{i j m l}\right) \frac{E^{2}(k)}{k^{4}} \chi_{k}(\sigma+\omega) \chi_{k}(\omega)
\end{aligned}
$$

where

$$
\begin{aligned}
T^{i j l m} & =\left(\delta^{i l}-\frac{k^{i} k^{l}}{k^{2}}\right)\left(\delta_{j m}-\frac{k^{j} k^{m}}{k^{2}}\right) \\
b_{i j} & \equiv \boldsymbol{e}_{i} \cdot\left(\nabla_{0}: \boldsymbol{\xi}\right) \cdot \boldsymbol{e}_{j},
\end{aligned}
$$

where the double dot denotes the tensor product.

To compute the coefficients $b_{i j}$ in Eq. (A.3), we follow the procedure derived by Belkacem et al. (2008), which infers that

$$
\begin{aligned}
& b_{r r}=\sum_{\ell, m}\left\{\left(\frac{\mathrm{d} \xi_{\mathrm{r}}}{\mathrm{d} r}\right) Y_{\ell, m}\right\}, \\
& b_{r \theta}=\sum_{\ell, m}\left\{\left(\frac{\mathrm{d} \xi_{\mathrm{H}}}{\mathrm{d} r}\right) \frac{\partial Y_{\ell, m}}{\partial \theta}+\left(\frac{\mathrm{d} \xi_{\mathrm{T}}}{\mathrm{d} r}\right) \frac{1}{\sin \theta} \frac{\partial Y_{\ell, m}}{\partial \theta}\right\}, \\
& b_{r \phi}=\sum_{\ell, m}\left\{\left(\frac{\mathrm{d} \xi_{\mathrm{H}}}{\mathrm{d} r}\right) \frac{1}{\sin \theta} \frac{\partial Y_{\ell, m}}{\partial \phi}-\left(\frac{\mathrm{d} \xi_{\mathrm{T}}}{\mathrm{d} r}\right) \frac{\partial Y_{\ell, m}}{\partial \theta}\right\}, \\
& b_{\theta r}=\sum_{\ell, m}\left\{\frac{1}{r}\left(\xi_{\mathrm{r}}-\xi_{\mathrm{H}}\right) \frac{\partial Y_{\ell, m}}{\partial \theta}-\frac{\xi_{\mathrm{T}}}{r} \frac{1}{\sin \theta} \frac{\partial Y_{\ell}^{m}}{\partial \phi}\right\}, \\
& b_{\theta \theta}=\sum_{\ell, m}\left\{\frac{\xi_{\mathrm{H}}}{r}\left(\frac{\partial^{2} Y_{\ell, m}}{\partial \theta^{2}}\right)+\frac{\xi_{\mathrm{r}}}{r} Y_{\ell, m}+\frac{\xi_{\mathrm{T}}}{r} \frac{\partial}{\partial \theta}\left(\frac{1}{\sin \theta} \frac{\partial Y_{\ell}^{m}}{\partial \phi}\right)\right\},
\end{aligned}
$$

$$
\begin{aligned}
b_{\theta \phi}= & \sum_{\ell, m}\left\{\frac{\xi_{\mathrm{H}}}{r} \frac{\partial}{\partial \theta}\left[\frac{1}{\sin \theta} \frac{\partial Y_{\ell, m}}{\partial \phi}\right]-\frac{\xi_{\mathrm{T}}}{r} \frac{\partial^{2} Y_{\ell}^{m}}{\partial \theta^{2}}\right\} \\
b_{\phi \theta}= & \sum_{\ell, m}\left\{\frac{\xi_{\mathrm{H}}}{r} \frac{\partial}{\partial \theta}\left[\frac{1}{\sin \theta} \frac{\partial Y_{\ell, m}}{\partial \phi}\right]+\frac{\xi_{\mathrm{T}}}{r}\left(\frac{\partial^{2} Y_{\ell}^{m}}{\partial \phi^{2}}+\frac{\cos \theta}{\sin \theta} \frac{\partial Y_{\ell}^{m}}{\partial \theta}\right)\right\}, \\
b_{\phi r}= & \sum_{\ell, m}\left\{\frac{1}{r}\left(\xi_{\mathrm{r}}-\xi_{\mathrm{H}}\right) \frac{1}{\sin \theta} \frac{\partial Y_{\ell, m}}{\partial \phi}+\frac{\xi_{\mathrm{T}}}{r} \frac{\partial Y_{\ell}^{m}}{\partial \theta}\right\}, \\
b_{\phi \phi}= & \sum_{\ell, m}\left\{\frac{\xi_{\mathrm{r}}}{r} Y_{\ell, m}+\frac{\xi_{\mathrm{H}}}{r}\left[\frac{1}{\sin ^{2} \theta}\left(\frac{\partial^{2} Y_{\ell, m}}{\partial \phi^{2}}\right)+\frac{\cos \theta}{\sin \theta}\left(\frac{\partial Y_{\ell, m}}{\partial \theta}\right)\right]\right. \\
& \left.+\frac{\xi_{\mathrm{T}}}{r}\left(\frac{\cos \theta}{\sin ^{2} \theta} \frac{\partial Y_{\ell}^{m}}{\partial \phi}-\frac{1}{\sin \theta} \frac{\partial^{2} Y_{\ell}^{m}}{\partial \phi \partial \theta}\right)\right\}
\end{aligned}
$$

The contribution of the Reynolds stress can thus be written as:

$$
\begin{aligned}
C_{\mathrm{R}}^{2}= & 4 \pi^{3} \int \mathrm{d} m \int \mathrm{d} k \int \mathrm{d} \omega R(r, k) \\
& \times \frac{E^{2}(k)}{k^{2}} \chi_{k}(\omega+\sigma) \chi_{k}(\omega)
\end{aligned}
$$

where we have defined $\mathrm{d} m=4 \pi r^{2} \rho_{0} \mathrm{~d} r$. Using the Einstein summation convention

$R(r, k)=\int \frac{\mathrm{d} \bar{\Omega}}{4 \pi} \int \frac{\mathrm{d} \Omega_{k}}{4 \pi} b_{i j}^{*} b_{l m}\left(T^{i j l m}+T^{i j m l}\right)$.

Because $T^{i j l m}=T^{j i m l}$, it is easy to show that

$R(r, k)=\int \frac{\mathrm{d} \bar{\Omega}}{4 \pi} \int \frac{\mathrm{d} \Omega_{k}}{4 \pi} B_{i j}^{*} B_{l m}\left(T^{i j l m}+T^{i j m l}\right)$,

where $B_{i j} \equiv(1 / 2)\left(b_{i j}+b_{j i}\right)$.

Using the expression Eq. (A.4) for $T^{i j l m}$, we write

$R(r, k)=R_{1}-R_{2}+R_{3}$

where

$$
\begin{aligned}
& R_{1}=2 \int \frac{\mathrm{d} \bar{\Omega}}{4 \pi} \int \frac{\mathrm{d} \Omega_{k}}{4 \pi}\left(\sum_{i, j} B_{i j}^{*} B_{i j}\right), \\
& R_{2}=4 \int \frac{\mathrm{d} \bar{\Omega}}{4 \pi} \int \frac{\mathrm{d} \Omega_{k}}{4 \pi}\left(\sum_{i, j} B_{i j}^{*} B_{i l} \frac{k_{j} k_{l}}{k^{2}}\right), \\
& R_{3}=2 \int \frac{\mathrm{d} \bar{\Omega}}{4 \pi} \int \frac{\mathrm{d} \Omega_{k}}{4 \pi}\left(\sum_{i, j} B_{i j}^{*} B_{l m} \frac{k_{i} k_{j} k_{l} k_{m}}{k^{4}}\right) .
\end{aligned}
$$

We assume isotropic turbulence, hence the $\boldsymbol{k}$ components satisfy

$\int \mathrm{d} \Omega_{k} \frac{k_{i} k_{j}}{k^{2}}=\delta_{i j} \int \mathrm{d} \Omega_{k} \frac{k_{\mathrm{r}}^{2}}{k^{2}}$,

where $\delta_{i j}$ is the Kronecker symbol for $i, j=r, \theta, \phi$. As in Belkacem et al. (2008), we then obtain

$$
\begin{aligned}
& R_{1}=2 \int \frac{\mathrm{d} \bar{\Omega}}{4 \pi}\left(\sum_{i, j}\left|B_{i j}\right|^{2}\right) \\
& R_{2}=2 \alpha R_{1}, \\
& R_{3}=\beta R_{1}+2 \beta\left(\int \frac{\mathrm{d} \bar{\Omega}}{4 \pi} \sum_{i \neq j}\left(B_{i i}^{*} B_{j j}+\text { c.c. }\right)\right),
\end{aligned}
$$


where we have set

$\alpha \equiv \int \frac{\mathrm{d} \Omega_{k}}{4 \pi} \frac{k_{\mathrm{r}}^{2}}{k^{2}} \quad$ and $\quad \beta \equiv \int \frac{\mathrm{d} \Omega_{k}}{4 \pi} \frac{k_{\mathrm{r}}^{4}}{k^{4}}$.

Using Eq. (A.6) to compute Eq. (A.8), with $\alpha=1 / 3$ and $\beta=1 / 5$ (see Belkacem et al. 2008, for details), yields

$$
\begin{aligned}
R(r)= & \frac{16}{15}\left|\frac{\mathrm{d} \xi_{\mathrm{r}}}{\mathrm{d} r}\right|^{2}+\frac{44}{15}\left|\frac{\xi_{\mathrm{r}}}{r}\right|^{2}+\frac{4}{5}\left(\frac{\xi_{\mathrm{r}}^{*}}{r} \frac{\mathrm{d} \xi_{\mathrm{r}}}{\mathrm{d} r}+\text { c.c. }\right) \\
& +L^{2}\left[\frac{11}{15}\left(|\mathcal{A}|^{2}+|\mathcal{B}|^{2}\right)-\frac{22}{15}\left(\frac{\xi_{\mathrm{r}}^{*} \xi_{\mathrm{H}}}{r^{2}}+\text { c.c. }\right)\right] \\
& +\left|\frac{\xi_{\mathrm{H}}}{r}\right|^{2}\left(\frac{16}{15} L^{4}+\frac{8}{5} \mathcal{F}_{\ell,|m|}-\frac{2}{3} L^{2}\right) \\
& +\left|\frac{\xi_{\mathrm{T}}}{r}\right|^{2}\left(\frac{11}{5} L^{2}\left(L^{2}-2\right)-\frac{8}{5} \mathcal{F}_{\ell,|m|}-\frac{2}{3} L^{2}\right) \\
& -\frac{2}{5} L^{2}\left(\frac{\mathrm{d} \xi_{\mathrm{r}}^{*}}{\mathrm{~d} r} \frac{\xi_{\mathrm{H}}}{r}+\text { c.c. }\right),
\end{aligned}
$$

where we have defined

$\mathcal{A}=\frac{\mathrm{d} \xi_{\mathrm{H}}}{\mathrm{d} r}+\frac{1}{r}\left(\xi_{\mathrm{r}}-\xi_{\mathrm{H}}\right) \quad$ and $\quad \mathcal{B}=\frac{\mathrm{d} \xi_{\mathrm{T}}}{\mathrm{d} r}-\frac{\xi_{\mathrm{T}}}{r}$

while

$\mathcal{F}_{\ell,|m|}=\frac{|m|(2 \ell+1)}{2}\left[\ell(\ell+1)-\left(m^{2}+1\right)\right]$

with $L^{2}=\ell(\ell+1)$.

\section{Appendix B: First-order perturbation of the excitation rates}

We recall the main results about the first-order perturbation of a spheroidal mode $\xi^{(0)}$ due to the Coriolis acceleration (of frequency $\omega_{0}$ ) and establish the perturbation of the excitation rates. Following the classical method given in Unno et al. (1989), we obtain

$\xi_{\mathrm{r}}=\xi_{\mathrm{r} ; n, l}^{(0)}+\left(\frac{2 \Omega}{\omega_{0}}\right) m \sum_{n^{\prime} \neq n} C_{n^{\prime}, n, l} \xi_{\mathrm{r} ; n^{\prime}, l^{\prime}}^{(0)}$,

$\xi_{\mathrm{H}}=\xi_{\mathrm{H} ; n, l}^{(0)}+\left(\frac{2 \Omega}{\omega_{0}}\right) m \sum_{n^{\prime} \neq n} C_{n^{\prime}, n, l} \xi_{\mathrm{H} ; n^{\prime}, l}^{(0)}$

and

$\xi_{\mathrm{T}}=i\left(\frac{2 \Omega}{\omega_{0}}\right) z_{m}^{l}$

where $\xi_{\mathrm{r} ; n^{\prime}, l}^{(0)}, \xi_{\mathrm{H} ; n^{\prime}, l}^{(0)}$ are solutions of the oscillation equation without rotation (Unno et al. 1989),

$$
\begin{aligned}
& C_{n^{\prime}, n, l}=\frac{\omega_{0}^{2}}{\left(\omega_{0}^{2}-\omega_{0 ; n^{\prime}}^{2}\right) I_{n^{\prime}}} \\
& \times \int_{0}^{R}\left[\xi_{\mathrm{H} ; n, l}^{(0)} \xi_{\mathrm{r} ; n^{\prime}, l}^{(0) *}+\left(\xi_{\mathrm{r} ; n, l}^{(0)}+\xi_{\mathrm{H} ; n, l}^{(0)}\right) \xi_{\mathrm{H} ; n^{\prime}, l}^{(0) *}\right] \rho_{0} r^{2} \mathrm{~d} r
\end{aligned}
$$

and

$$
\begin{aligned}
z_{l, m}= & l D_{l, m}\left[\xi_{\mathrm{r} ; n, l-1}^{(0)}-(l-1) \xi_{\mathrm{H} ; n, l-1}^{(0)}\right] \\
& -(l+1) D_{l+1, m}\left[\xi_{\mathrm{r} ; n, l+1}^{(0)}+(l+2) \xi_{\mathrm{H} ; n, l+1}^{(0)}\right],
\end{aligned}
$$

where

$D_{l, m}=\frac{1}{l^{2}} \sqrt{\frac{l^{2}-m^{2}}{4 l^{2}-1}}$.

The Coriolis corrective terms are of the order of $2 \Omega / \omega_{0}$, which is here assumed to be small. Inserting Eqs. (B.1)-(B.3) into Eq. (15), we obtain

$C_{\mathrm{R}, m}^{2}=\left[C_{\mathrm{R}}^{(0)}\right]^{2}+m\left(\frac{2 \Omega}{\omega_{0}}\right)\left[C_{\mathrm{R},|m|}^{(1)}\right]^{2}$

where

$$
\begin{aligned}
& {\left[C_{\mathrm{R}}^{(0)}\right]^{2}=4 \pi^{3} \int \mathrm{d} m R^{(0)} S_{R}\left(\omega_{0}\right),} \\
& {\left[C_{\mathrm{R},|m|}^{(1)}\right]^{2}=4 \pi^{3} \int \mathrm{d} m R_{|m|}^{(1)} S_{R}\left(\omega_{0}\right),}
\end{aligned}
$$

and $R^{(0)}$ and $R_{|m|}^{(1)}$ correspond to the perturbative expansion of $R$ given in Eq. (16) where

$$
\begin{aligned}
R^{(0)}= & \frac{16}{15}\left|\frac{\mathrm{d} \xi_{\mathrm{r} ; n, l}^{(0)}}{\mathrm{d} r}\right|^{2}+\frac{44}{15}\left|\frac{\xi_{\mathrm{r} ; n, l}^{(0)}}{r}\right|^{2}+\frac{4}{5}\left(\frac{\xi_{\mathrm{r} ; n, l}^{(0) *}}{r} \frac{\mathrm{d} \xi_{\mathrm{r} ; n, l}^{(0)}}{\mathrm{d} r}+\text { c.c. }\right) \\
& +L^{2}\left[\frac{11}{15}\left(|\mathcal{A}|^{2}+|\mathcal{B}|^{2}\right)-\frac{22}{15}\left(\frac{\xi_{\mathrm{r} ; n, l}^{(0) *} \xi_{\mathrm{H} ; n, l}^{(0)}}{r^{2}}+\text { c.c. }\right)\right] \\
& +\left|\frac{\xi_{\mathrm{H} ; n, l}^{(0)}}{r}\right|^{2}\left(\frac{16}{15} L^{4}+\frac{8}{5} \mathcal{F}_{\ell,|m|}-\frac{2}{3} L^{2}\right) \\
& -\frac{2}{5} L^{2}\left(\frac{\mathrm{d} \xi_{\mathrm{r} ; n, l}^{(0) *}}{\mathrm{~d} r} \frac{\xi_{\mathrm{H} ; n, l}^{(0)}}{r}+\text { c.c. }\right), \\
R^{(1)}= & \sum_{n^{\prime} \neq n} C_{n^{\prime}, n, l} f_{n^{\prime}, n, l,|m|}
\end{aligned}
$$

where

$$
\begin{aligned}
& f_{n^{\prime}, n, l,|m|}=\frac{16}{15}\left[\frac{\mathrm{d} \xi_{\mathrm{r} ; n, l}^{(0)}}{\mathrm{d} r} \frac{\mathrm{d} \xi_{\mathrm{r} ; n^{\prime}, l}^{(0) *}}{\mathrm{~d} r}+\text { c.c. }\right] \\
& +\frac{44}{15}\left[\frac{\xi_{\mathrm{r} ; n, l}^{(0)} \xi_{\mathrm{r} ; n^{\prime}, l}^{(0) *}}{r}+\text { c.c. }\right] \\
& +\left(\frac{16}{15} L^{4}-\frac{2}{3} L+\frac{8}{5} \mathcal{F}_{l,|m|}\right)\left[\frac{\xi_{\mathrm{H} ; n, l}^{(0)} \xi_{\mathrm{H} ; n^{\prime}, l}^{(0) *}}{r^{2}}+\text { c.c. }\right] \\
& +\frac{11}{15} L^{2}\left[\left(\frac{\mathrm{d} \xi_{\mathrm{H} ; n, l}^{(0)}}{\mathrm{d} r}+\frac{\xi_{\mathrm{r}, n, l}^{(0)}-\xi_{\mathrm{H} ; n, l}^{(0)}}{r^{2}}\right)\right. \\
& \left.\times\left(\frac{\mathrm{d} \xi_{\mathrm{H} ; n^{\prime}, l}^{(0) *}}{\mathrm{~d} r}+\frac{\xi_{\mathrm{r} ; n^{\prime}, l}^{(0) *}-\xi_{\mathrm{H} ; n^{\prime}, l}^{(0) *}}{r^{2}}\right)+\text { c.c. }\right] \\
& +\frac{4}{5}\left(\frac{\mathrm{d} \xi_{\mathrm{r} ; n, l}^{(0)}}{\mathrm{d} r} \frac{\xi_{\mathrm{r} ; n^{\prime}, l}^{(0) *}}{r}+\frac{\mathrm{d} \xi_{\mathrm{r} ; n^{\prime}, l}^{(0)}}{\mathrm{d} r} \frac{\xi_{\mathrm{r} ; n, l}^{(0) *}}{r}+\text { c.c. }\right) \\
& -\frac{2}{5} L\left(\frac{\xi_{\mathrm{H} ; n, l}^{(0)}}{r} \frac{\mathrm{d} \xi_{\mathrm{r} ; n^{\prime}, l}^{(0) *}}{\mathrm{~d} r}+\frac{\xi_{\mathrm{H} ; n^{\prime}, l}^{(0)}}{r} \frac{\mathrm{d} \xi_{\mathrm{r} ; n, l}^{(0) *}}{\mathrm{~d} r}+\text { c.c. }\right) \\
& -\frac{22}{15} L\left(\frac{\xi_{\mathrm{r} ;, l,}^{(0)} \xi_{\mathrm{H} ; n^{\prime}, l}^{(0) *}}{r}+\frac{\xi_{\mathrm{r} ; n^{\prime}, \xi^{(0)}, \xi^{2}, l}^{(0) *}}{r}+\text { c.c. }\right) \text {. }
\end{aligned}
$$


Similarly, we get for the entropic term given in Eq. (21)

$C_{S}^{2}=\left[C_{S}^{(0)}\right]^{2}+\left(\frac{2 \Omega}{\omega_{0 ; n, l}}\right) m\left[C_{S}^{(1)}\right]^{2}$

where

$$
\left[C_{S}^{(0)}\right]^{2}=\frac{4 \pi^{3} \mathcal{H}}{\omega_{0 ; n, l}^{2}} \int \mathrm{d}^{3} x_{0} \alpha_{s}\left(A_{l}^{(0)}+B_{l}^{(0)}\right) S_{S}\left(\omega_{0 ; n, l}\right)
$$

and

$$
\left[C_{S}^{(1)}\right]^{2}=\frac{4 \pi^{3} \mathcal{H}}{\omega_{0 ; n, l}^{2}} \int \mathrm{d}^{3} x_{0} \alpha_{s}\left(A_{l}^{(1)}+B_{l}^{(1)}\right) S_{S}\left(\omega_{0 ; n, l}\right) .
$$

We have

$$
\begin{aligned}
A_{l}^{(0)}= & \frac{1}{r^{2}}\left|D_{l}^{(0)} \frac{\mathrm{d} \ln \left|\alpha_{s}\right|}{\mathrm{d} \ln r}-\frac{\mathrm{d} D_{l}^{(0)}}{\mathrm{d} \ln r}\right|^{2} \\
A_{l}^{(1)}= & \frac{1}{r^{2}}\left(D_{l}^{(0)} \frac{\mathrm{d} \ln \left|\alpha_{s}\right|}{\mathrm{d} \ln r}-\frac{\mathrm{d} D_{l}^{(0)}}{\mathrm{d} \ln r}\right) \\
& \times \sum_{n^{\prime}}\left\{C_{n^{\prime}, n, l, m}\left(D_{l}^{(0) *}\left(n^{\prime}\right) \frac{\mathrm{d}\left|\alpha_{s}\right|}{\mathrm{d} \ln r}-\frac{\mathrm{d} D_{l}^{(0) *}}{\mathrm{~d} \ln r}\right)\right\}+\text { c.c., }
\end{aligned}
$$

$B_{l}^{(0)}=\frac{1}{r^{2}} L^{2}\left|D_{l}^{(0)}\right|^{2}$

and

$B_{l}^{(1)}=\frac{1}{r^{2}} L^{2} D_{l}^{(0)} \sum_{n^{\prime}} C_{n^{\prime}, n, l, m} D_{l}^{(0) *}\left(n^{\prime}\right)+$ c.c.

where

$D_{l}=\frac{1}{r^{2}} \frac{\mathrm{d}\left(r^{2} \xi_{\mathrm{r} ; n, l, m}^{(0)}\right)}{\mathrm{d} r}-\frac{L^{2}}{r} \xi_{\mathrm{H} ; n, l, m}^{(0)}$.

\section{References}

Appourchaux, T., Fröhlich, C., Andersen, B., et al. 2000, ApJ, 538, 401 Appourchaux, T., Michel, E., Auvergne, M., et al. 2008, A\&A, 488, 705 Auvergne, M., Bodin, P., Boisnard, L., et al. 2009, A\&A, 506, 411
Balmforth, N. J. 1992, MNRAS, 255, 639

Belkacem, K., Samadi, R., Goupil, M. J., \& Kupka, F. 2006a, A\&A, 460, 173 Belkacem, K., Samadi, R., Goupil, M. J., Kupka, F., \& Baudin, F. 2006b, A\&A, 460,183

Belkacem, K., Samadi, R., Goupil, M.-J., \& Dupret, M.-A. 2008, A\&A, 478, 163 Belkacem, K., Samadi, R., Goupil, M. J., et al. 2009, A\&A, 494, 191

Chaplin, W. J., Appourchaux, T., Arentoft, T., et al. 2008, Astron. Nachr., 329, 549

Chaplin, W. J., Houdek, G., Elsworth, Y., et al. 2005, MNRAS, 360, 859

Christensen-Dalsgaard, J., Arentoft, T., Brown, T. M., et al. 2008, Commun. Asteroseismol., 157, 266

Decressin, T., Mathis, S., Palacios, A., et al. 2009, A\&A, 495, 271

Dintrans, B., \& Rieutord, M. 2000, A\&A, 354, 86

Dziembowski, W. A., \& Goode, P. R. 1992, ApJ, 394, 670

Espinosa Lara, F., \& Rieutord, M. 2007, A\&A, 470, 1013

Gabriel, A. H., Baudin, F., Boumier, P., et al. 2002, A\&A, 390, 1119

García, R. A., Jiménez, A., Mathur, S., et al. 2008a, Astron. Nachr., 329, 476

García, R. A., Mathur, S., \& Ballot, J. 2008b, Sol. Phys., 251, 135

García, R. A., Turck-Chièze, S., Jiménez-Reyes, S. J., et al. 2007, Science, 316, 1591

Goldreich, P., \& Keeley, D. A. 1977, ApJ, 212, 243

Goldreich, P., Murray, N., \& Kumar, P. 1994, ApJ, 424, 466

Goupil, M., Deheuvels, S., Provost, J., et al. 2009, A\&A, submitted

Houdek, G. 2006, in SOHO 18/GONG 2006/HeIAS I: beyond the spherical Sun, ed. K. Fletcher, ESA SP-624, Noordwijk [arXiv: astro-ph/0612024]

Kumar, P., Narayan, R., \& Loeb, A. 1995, ApJ, 453, 480

Leibacher, J. W., \& Stein, R. F. 1971, Astrophys. Lett., 7, 191

Maeder, A. 2009, Physics, Formation and Evolution of Rotating Stars (Berlin, Heidelberg: Springer), Astron. Astrophys. Library

Maeder, A., \& Meynet, G. 2000, ARA\&A, 38, 143

Mathis, S., Talon, S., Pantillon, F.-P., \& Zahn, J.-P. 2008, Sol. Phys., 251, 101

Mathur, S., Eff-Darwich, A., García, R. A., \& Turck-Chièze, S. 2008, A\&A, 484, 517

Mathur, S., Turck-Chièze, S., Couvidat, S., \& García, R. A. 2007, ApJ, 668, 594 Michel, E., Baglin, A., Auvergne, M., et al. 2008a, Science, 322, 558

Michel, E., Baglin, A., Weiss, W. W., et al. 2008b, Commun. Asteroseismol., 157,69

Miesch, M. S. 2005, Liv. Rev. Sol. Phys., 2, 1

Reese, D., Lignières, F., \& Rieutord, M. 2006, A\&A, 455, 621

Rieutord, M., \& Valdettaro, L. 1997, J. Fluid Mech., 341, 77

Samadi, R., \& Goupil, M. J. 2001, A\&A, 370, 136

Samadi, R., Ludwig, H., Belkacem, K., et al. 2009, A\&A, in press [arXiv: 0910.4027]

Samadi, R., Nordlund, Å., Stein, R. F., Goupil, M. J., \& Roxburgh, I. 2003, A\&A, 403, 303

Stein, R., Georgobiani, D., Trampedach, R., Ludwig, H.-G., \& Nordlund, Å. 2004, Sol. Phys., 220, 229

Stein, R. F. 1967, Sol. Phys., 2, 385

Talon, S., Zahn, J.-P., Maeder, A., \& Meynet, G. 1997, A\&A, 322, 209

Turck-Chièze, S., García, R. A., Couvidat, S., et al. 2004, ApJ, 604, 455

Ulrich, R. K. 1970, ApJ, 162, 993

Unno, W., Osaki, Y., Ando, H., Saio, H., \& Shibahashi, H. 1989, Nonradial oscillations of stars (University of Tokyo Press), 2nd edn. 